\title{
Novel Use of Hypoxia-Inducible Polymerizable Protein to Augment Chemotherapy for Pancreatic Cancer
}

\author{
Andrew Gdowski ${ }^{1}$, Hamed Hayatshahi ${ }^{2}\left(\mathbb{0}\right.$, Rafal Fudala $^{3}$, Rohan Joshi ${ }^{3}$, Jin Liu ${ }^{4}$, Jamboor K. Vishwanatha ${ }^{3}$, \\ Rohan Jeyarajah ${ }^{5}$, Paul Guzik ${ }^{6}$ and Amalendu P. Ranjan ${ }^{3, *}$
}

1 Lineberger Comprehensive Cancer Center, University of North Carolina, Chapel Hill, NC 27599, USA; andrewgdowski@gmail.com

2 Reata Pharmaceuticals Inc., Plano, TX 75024, USA; Hamed.Hayatshahi@reatapharma.com

3 Department of Microbiology, Immunology and Genetics, University of North Texas Health Science Center, Fort Worth, TX 76107, USA; Rafal.fudala@unthsc.edu (R.F.); Rohan.Joshi@my.unthsc.edu (R.J.); Jamboor.vishwanatha@unthsc.edu (J.K.V.)

4 Department of Pharmaceutical Sciences, University of North Texas System College of Pharmacy, University of North Texas Health Science Center, Fort Worth, TX 76107, USA; Jin.Liu@unthsc.edu

5 TCU and UNTHSC School of Medicine, Fort Worth, TX 76107, USA; rohanjeyarajah@gmail.com

6 Division of Gastroenterology \& Hepatology, Loma Linda University Medical Center, Loma Linda, CA 92354, USA; paul.r.guzik@gmail.com

* Correspondence: amalendu.ranjan@unthsc.edu; Tel.: +1-817-735-2138

\section{check for}

updates

Citation: Gdowski, A.;

Hayatshahi, H.; Fudala, R.; Joshi, R.; Liu, J.; Vishwanatha, J.K.;

Jeyarajah, R.; Guzik, P.; Ranjan, A.P. Novel Use of Hypoxia-Inducible

Polymerizable Protein to Augment

Chemotherapy for Pancreatic Cancer.

Pharmaceutics 2022, 14, 128.

https://doi.org/10.3390/

pharmaceutics14010128

Academic Editor: Santosh Aryal

Received: 2 December 2021

Accepted: 31 December 2021

Published: 5 January 2022

Publisher's Note: MDPI stays neutral with regard to jurisdictional claims in published maps and institutional affiliations.

Copyright: (c) 2022 by the authors. Licensee MDPI, Basel, Switzerland. This article is an open access article distributed under the terms and conditions of the Creative Commons Attribution (CC BY) license (https:/ / creativecommons.org/licenses/by/ $4.0 /)$.

\begin{abstract}
Pancreatic ductal adenocarcinoma (PDAC) is one of the most aggressive malignancies and is the fourth leading cause of cancer-related deaths in the United States. Unfortunately, 80-85\% of patients are diagnosed with unresectable, advanced stage tumors. These tumors are incurable and result in a median survival less than approximately six months and an overall 5-year survival rate of less than $7 \%$. Whilst chemotherapy is a critical treatment, cure is not possible without surgical resection. The poor clinical outcomes in PDAC can be partially attributed to its dense desmoplastic stroma, taking up roughly $80 \%$ of the tumor mass. The stroma surrounding the tumor disrupts the normal architecture of pancreatic tissue leading to poor vascularization, high intratumoral pressure along with hypoxia and an acidic tumor microenvironment. This complicated microenvironment presents a significant challenge for drug delivery. The current manuscript discusses a novel approach to overcome many of these various obstacles. A complex of gemcitabine (GEM) and hemoglobin S $(\mathrm{HbS})$ was formulated, which self-polymerizes under hypoxic and acidic conditions. When polymerized, $\mathrm{HbS}$ has the potential to break the tumor stroma, decrease intratumoral pressure, and therefore improve the treatment efficacy of standard therapy. Intratumoral injection of $\mathrm{HbS}$ with a fluorescent small molecule surrogate for GEM into a pancreatic tumor xenograft resulted in improved dissemination of the small molecule throughout the pancreatic tumor. The self-polymerization of $\mathrm{HbS}+\mathrm{GEM}$ was significantly more effective than either agent individually at decreasing tumor size in an in vivo PDAC mouse model. These findings would suggest a clinical benefit from delivering the complex of GEM and $\mathrm{HbS}$ via direct injection by endoscopic ultrasound (EUS). With such a treatment option, patients with locally advanced disease would have the potential to become surgical candidates, offering them a chance for cure.
\end{abstract}

Keywords: hemoglobin S; pancreatic cancer; gemcitabine; polymerization; hypoxia

\section{Introduction}

Pancreatic ductal adenocarcinoma (PDAC) is one of the most lethal human malignancies with an overall 5-year survival rate below 7\% [1]. The majority of patients with PDAC are not candidates for surgical resection due to local tumor invasion and metastasis. Even with currently available systemic and radiation therapy options, overall survival is limited $[2,3]$. The antimetabolite small molecule gemcitabine (GEM) is one potential agent 
used to treat PDAC but results in only minimal increased survival. Despite recent advances with additional chemotherapy regimens, the efficacy of chemotherapy remains poor [4]. Increasing evidence shows that the pancreatic tumor microenvironment presents several barriers to treatment delivery. The major challenges are enriched tumor stromal components and a disorganized vasculature of pancreatic cancer tissues, which make it difficult to deliver a sufficient amount of chemotherapy into the tumor due to higher interstitial pressure [5,6]. Furthermore, insufficient tissue oxygenation, or hypoxia, contributes to solid tumor expansion [7]. Efforts have been made to develop and study drugs that target this novel pancreatic cancer microenvironment [8]. However, so far, these newer agents have been insufficient.

Sickle cell hemoglobin $(\mathrm{HbS})$ is a tetrameric protein and a variant of the normal hemoglobin protein in which a point mutation causes a substitution of valine for glutamic acid on the beta globin chain of the tetrameric molecule. The consequence of this substation is that when $\mathrm{HbS}$ molecules are in a deoxygenated or acidic state, there is a confirmation change in the protein that facilitates noncovalent interactions between the side chain residues of $B$ Val6 and the hydrophobic grooves of amino acid residues of a neighboring tetramer. This results in polymerization and precipitation of $\mathrm{HbS}$, which forms the classic sickled shape of red blood cells seen in sickle cell anemia $[9,10]$. In this paper, we tested the hypothesis that polymerization of $\mathrm{HbS}$ can be exploited in the hypoxic and acidic pancreatic tumor microenvironment to overcome the dense reactive tumor stroma and utilize this strategy to simultaneously distribute a small molecule throughout the tumor to decrease tumor size. For PDAC, direct injection into the tumor using interventional endoscopic ultrasonography (EUS) guided local therapy could easily be used to deliver this therapy directly to the tumor in a clinical setting [11] (Scheme 1).

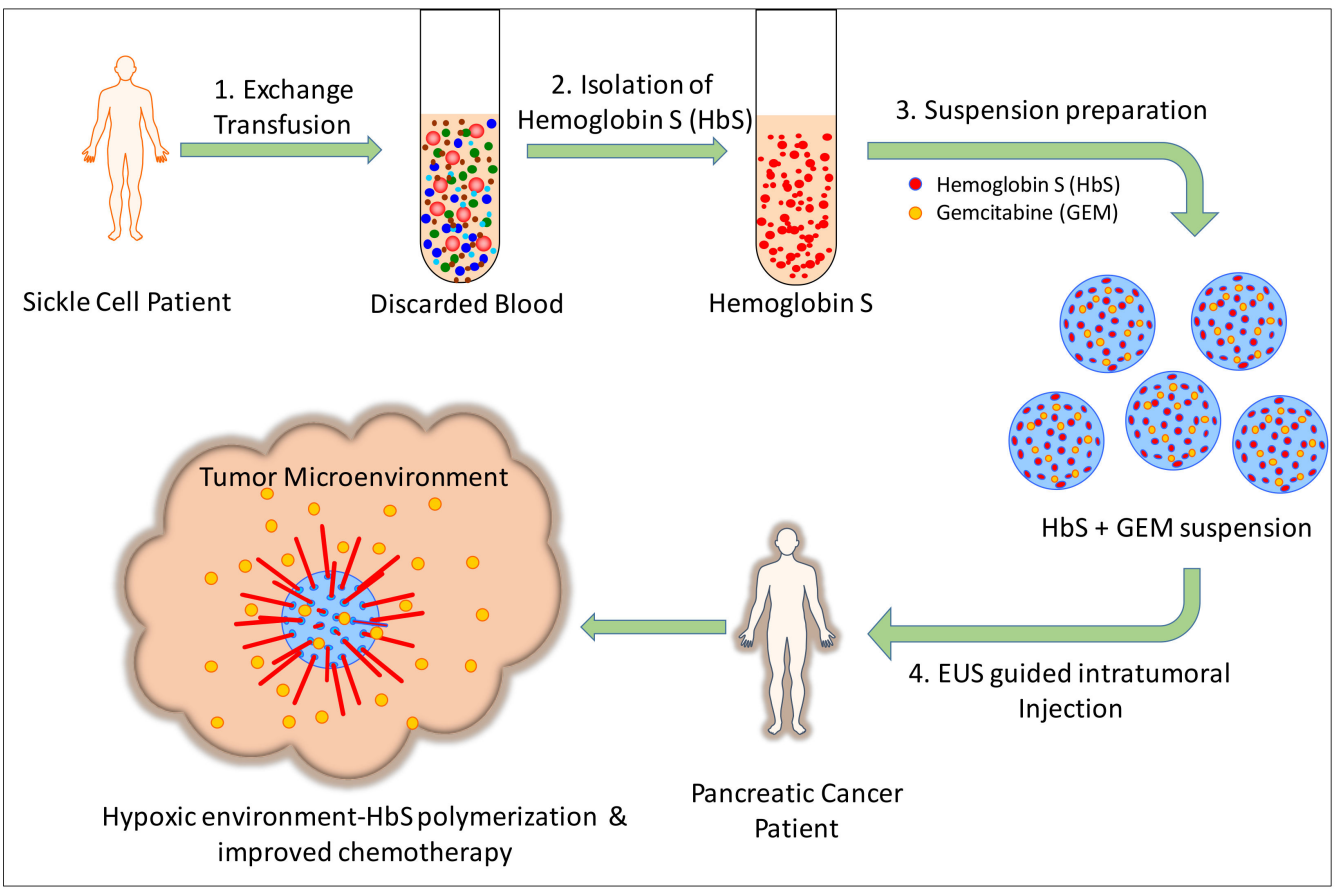

Scheme 1. Theoretical overview of hypoxia-induced polymerization of $\mathrm{HbS}$ and synergistic chemotherapy. (1.) Blood could be harvested during exchange transfusion from a patient with known sickle cell anemia or HbS could be engineered in a lab. (2.) HbS could be isolated and pooled together. (3.) $\mathrm{HbS}$ and GEM can be complexed in solution. (4.) An endoscopic ultrasound (EUS) injection can deliver therapy directly to the tumor. Once in the tumor microenvironment, $\mathrm{HbS}$ polymerizes and allows improved diffusion of GEM throughout the tumor. 


\section{Materials and Methods}

\subsection{Materials}

Gemcitabine hydrochloride (GEM.HCl) ( $\geq 98 \%$ ) was purchased from Proteochem, UT, USA. Hemoglobin $\mathrm{S}(\mathrm{HbS})$ was purchased from Sigma-Aldrich, St. Louis, MO, USA. RPMI 1640 medium, fetal bovine serum (FBS), and phosphonate buffered solution (PBS) were supplied from Hyclone, Marlborough, MA, USA. Cell culture procedures: BxPC3 cells used for animal studies were purchased from American Type Culture Collection (ATCC) and tested negative for mycoplasma contamination. Cells were grown in RPMI 1640 supplemented with $2 \mathrm{mM}$ L-glutamine and 10\% FBS and used for in vivo studies.

\subsection{Suspension Preparation}

$\mathrm{HbS}$ was suspended at a concentration of $20 \mathrm{mg} / \mathrm{mL}$ in PBS. Gemcitabine hydrochloride was next added into a solution by suspending in saline separately. The gemcitabine solution was added to the $\mathrm{HbS}$ solution to make the final suspension of gemcitabine and $\mathrm{HbS}$ that was then vortexed for $30 \mathrm{~s}$. For animal experiments, the solution was drawn up in $1 \mathrm{cc}, 30 \mathrm{G}$ syringes for injection. This was prepared immediately prior to use.

\subsection{In Silico Molecular Docking of HbS and GEM}

We prepared the PDB entries 1NEJ [12] and 2HBS [13] as the crystal structures of HbS in oxygenated and deoxygenated forms, respectively, via Autodock Tools [14]. With the same program, we also prepared GEM as a ligand. Then, using Autodock vina [15], we docked GEM onto both structures within a searching box that would encompass the whole protein to look for potential binding sites.

\subsection{Absorption Spectrum Analysis of $\mathrm{HbS}$}

UV-vis absorption spectra of $\mathrm{HbS}$ in PBS buffer were recorded using a Cary 60 Bio UV-visible spectrophotometer (Varian, Inc., Mulgrave, Australia). All measurements were performed at room temperature in $1 \mathrm{~cm} \times 1 \mathrm{~cm}$ cuvettes.

\subsection{In Vivo Drug Diffusion Study}

$\mathrm{HbS}$ solution was prepared as described above. IR-780 iodide dye (Sigma Aldrich) was diluted in DMSO and mixed with $\mathrm{HbS}$ solution by vortexing for $30 \mathrm{~s}$. For the $\mathrm{HbS}$ treatment group, a 1:1 (v/v) mixture of $\mathrm{HbS}$ solution with IR-780 dye solution was used. For the control group, a 1:1 (v/v) mixture of $\mathrm{HbS}$ solution with saline was used. A total of $100 \mu \mathrm{L}$ was injected intratumorally in each tumor after the tumor had developed for 21 days as described below. Imaging was performed at $10 \mathrm{~min}, 30 \mathrm{~min}$, and $1 \mathrm{~h}$ by IVIS live animal imaging machine.

\subsection{In Vivo Tumor Efficacy Study}

Animal procedures were performed according to protocols approved by the IACUC at the University of North Texas Health Science Center, Texas, USA. A subcutaneous xenograft tumor model was used to test in vivo drug and $\mathrm{HbS}$ synergistic efficacy. Four-to-six-weekold female athymic mice were donated from ENVIGO. Mice were allowed to acclimate for 1 week before experiments. Mice were injected with $1 \times 10^{6} \mathrm{BxPC} 3$ pancreatic cancer cells mixed 1:1 with Matrigel (BD Bioscience, San Jose, CA, USA) in a total volume of $0.2 \mathrm{~mL}$. This was implanted to the subcutaneous area of the right flank of the nude mouse. Tumors developed for 1 week, then were randomized into groups by determining the tumor size using vernier caliper so that the starting average size was equal across treatment groups. Treatment commenced on day 7 with weekly $0.100 \mathrm{~mL}$ intratumoral injections. For the treatment experiment, mice ( $n=7$ /group) bearing subcutaneous BXPC3 tumors were injected with saline, $\mathrm{HbS}(20 \mathrm{mg} / \mathrm{mL}$ ) alone, GEM (50 mg/ kg body weight) alone, and a combination of $\mathrm{HbS}$ and GEM (50 mg/ $\mathrm{kg}$ body weight) intratumorally. Tumor sizes were measured at least twice a week for 27 days. When the tumor reached the final time point, 
the mouse was euthanized by $\mathrm{CO}_{2}$ asphyxiation. Tumor volume $\left(\mathrm{mm}^{3}\right)$ was calculated as (length $\times$ width $\left.^{2}\right) / 2$.

\section{Results}

\subsection{In Silico Interaction Analysis of HbS and GEM}

In order to determine if there is an interaction between $\mathrm{HbS}$ and GEM molecules, we performed in silico docking of GEM on HbS. There was a significant interaction between GEM and multiple areas on the $\mathrm{HbS}$ subunits, although the highest affinities were predicted for binding poses between the $\alpha$ and $\beta$ chains (Figure 1). This interaction was modeled in both the oxygenated conformation and deoxygenated conformation of $\mathrm{HbS}$. It was found that the poses of GEM on the deoxygenated form of $\mathrm{HbS}$ had lower affinities compared to the oxygenated form. This implies that GEM can be loaded on $\mathrm{HbS}$ while in an oxygenated state. Then, when $\mathrm{HbS}$ is in a hypoxic condition such as the tumor microenvironment, not only will it start to polymerize, but it will also offload any GEM that is bound to the protein. This is an important finding in this proposed treatment as this essentially demonstrates delivery of a potent drug at the target site. This ensures reduced off target effects of the drug. It has not escaped our attention that this offloading of drug from the $\mathrm{HbS}$ during a conformational change could also serve a role in loading other small molecules on $\mathrm{HbS}$ and systemically delivering chemotherapy to other tumor sites.
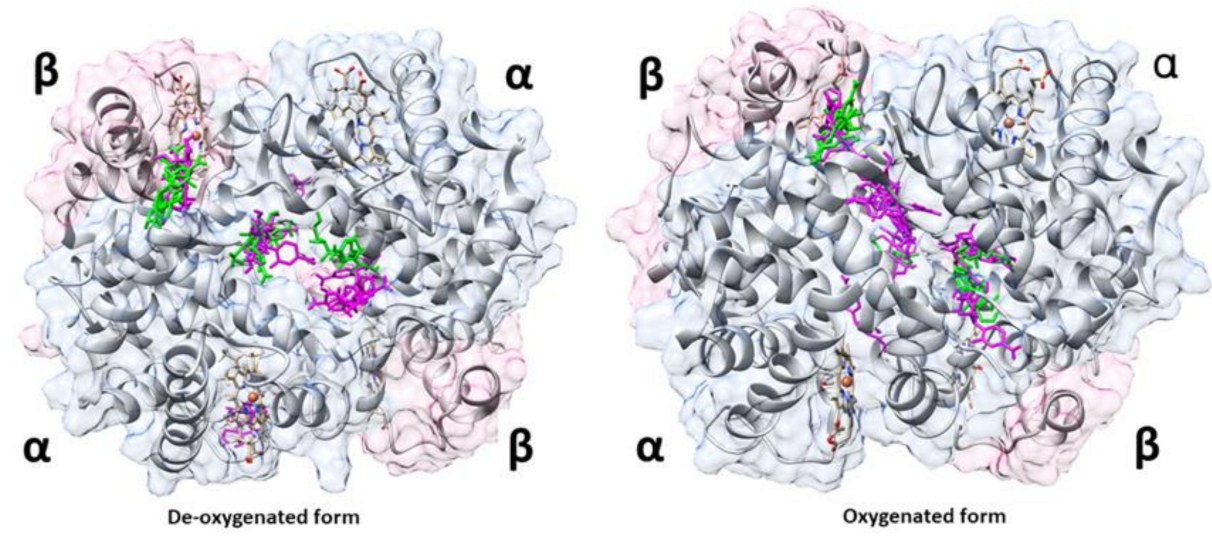

Figure 1. In silico molecular docking poses of gemcitabine on hemoglobin $S$ tetramer in deoxygenated (left) and oxygenated (right) forms. In the deoxygenated form (left), the green poses are poses with binding energy of -6.0 to $-6.4 \mathrm{kcal} / \mathrm{mol}$ and the pink poses are those with binding energies of -5.7 to $-5.9 \mathrm{kcal} / \mathrm{mol}$. In the oxygenated form (right), the green poses are poses with binding energy of -6.5 to $-6.9 \mathrm{kcal} / \mathrm{mol}$ and the pink poses are those with binding energies of -6.0 to $-6.4 \mathrm{kcal} / \mathrm{mol}$. The best poses in both forms are those between the $\alpha$ and $\beta$ chains. Figures were prepared with UCSF Chimera [16].

\subsection{Characterization of Polymerization of Hemoglobin S under Hypoxia}

It was suspected that the structural changes of $\mathrm{HbS}$ due to polymerization under hypoxic conditions might be reflected in the form of a variation in absorption [17]. Therefore, the absorption spectrum of the $\mathrm{HbS}$ protein was recorded and compared under hypoxia and normoxia conditions. The reason that a spectral change occurs is that chromophores residing within chemical/biochemical molecules have varying interactions with the environment depending on their conformation. When a change in conformation occurs within a molecule, the chromophores become more shielded or less shielded from solvent molecules. The outcome is a change in the spectral properties of the chromophores and absorption characteristics observed. The comparison of the $\mathrm{HbS}$ absorption spectra under hypoxia ( $1 \%$ oxygen), normoxia ( $21 \%$ oxygen), and hypoxia after $21 \%$ oxygen (normoxia condition) exposure is presented in Figure 2. The intensity of the absorption transition near $400 \mathrm{~nm}$ significantly increases with hypoxia (green arrow). Additionally, there are new features in the absorption spectrum under hypoxia near $550 \mathrm{~nm}$ and $650 \mathrm{~nm}$. Black arrows on Figure 2 
insert show the areas where the changes occurred. Next, after the hypoxia treatment, HbS was exposed to $21 \%$ oxygen (normoxia condition, $1 \mathrm{~h}$ at $37^{\circ} \mathrm{C}$ ) to determine if the observed differences in absorption spectra were permanent conformational changes or reversible. This re-exposure to oxygen did not revert back to the original absorption spectrum seen under oxygenated conditions (normoxia), suggesting the conformation change from hypoxia was permanent (Figure 2).

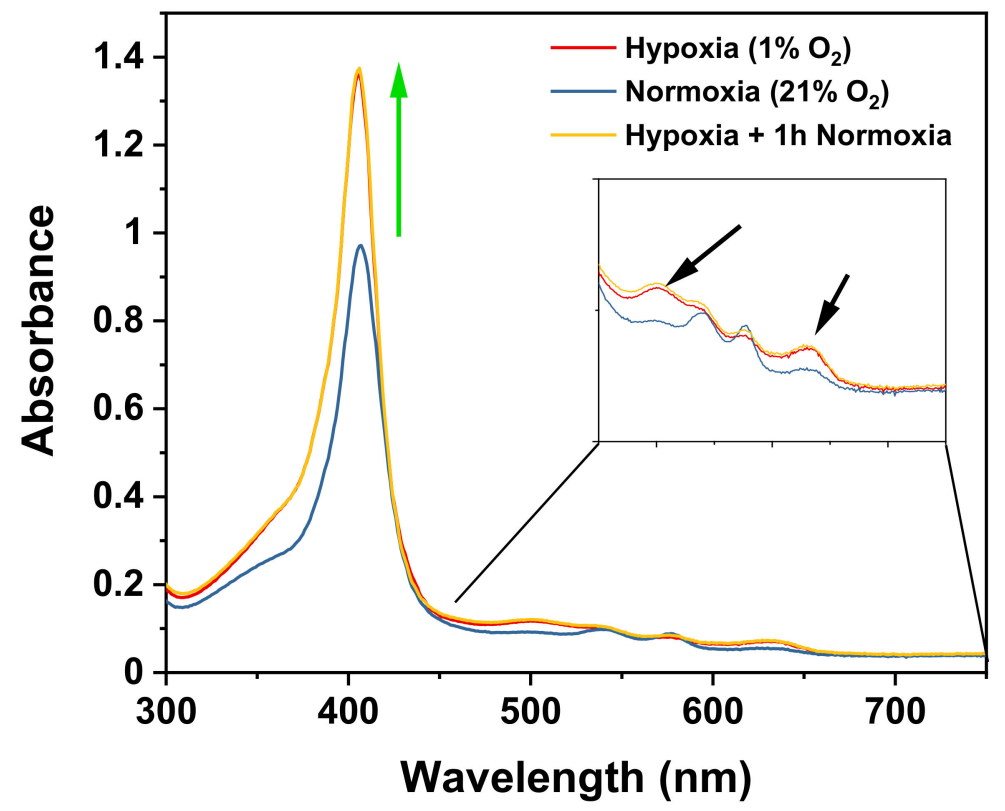

Figure 2. UV-vis absorption spectra of $\mathrm{HbS}$ protein after overnight treatment under hypoxia ( $1 \%$ oxygen), normoxia ( $21 \%$ oxygen), and after exposing the hypoxia samples to $21 \%$ oxygen (normoxia condition) at $37^{\circ} \mathrm{C}$ for $1 \mathrm{~h}$.

\subsection{In Vivo Drug Diffusion Tumor Model}

An IR dye was used as a surrogate molecule for GEM to determine the effect HbS polymerization has on the ability of drugs to diffuse throughout the tumor. The IR dye was chosen based on its small molecular weight and ability to easily detect a fluorescent signal during live animal imaging.

The tumors treated with $\mathrm{HbS}$ trended toward having a more ubiquitous distribution of dye throughout the tumor after injection at $10 \mathrm{~min}, 30 \mathrm{~min}$, and $1 \mathrm{~h}$. This supports the notion that $\mathrm{HbS}$ polymerization disrupts the tumor microenvironment and can improve diffusion of small molecules throughout the tumor (Figure 3).

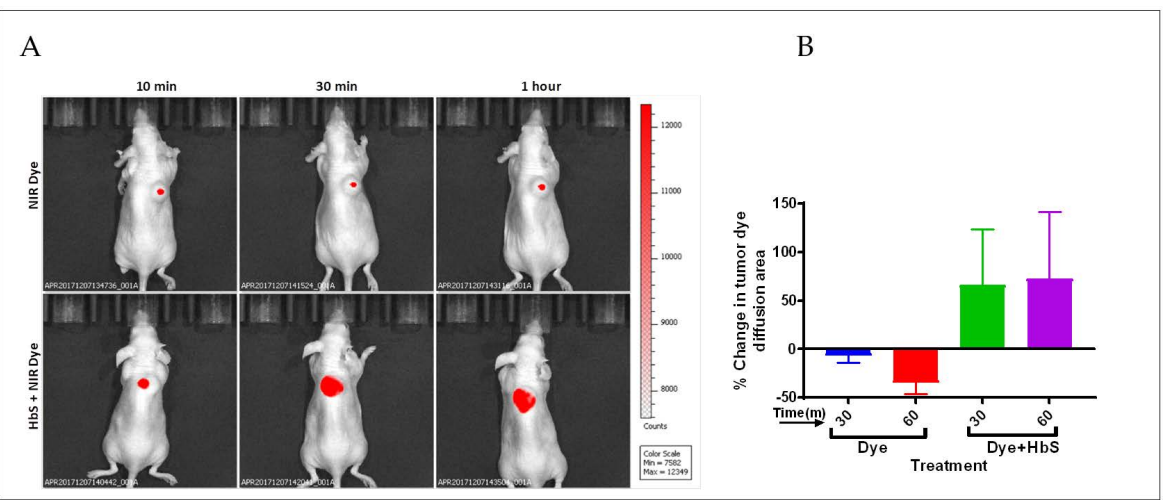

Figure 3. In vivo distribution of NIR dye (IR-780-I) in nude mice bearing xenografted BxPC3 pancreatic tumor at $10 \mathrm{~min}, 30 \mathrm{~min}$, and $1 \mathrm{~h}$ after intratumoral injection (A). Fluorescence quantification of dye distribution in tumors at $30 \mathrm{~min}$ and $1 \mathrm{~h} \mathrm{(B).}$ 


\subsection{In Vivo Efficacy in Pancreatic Tumor Model}

A subcutaneous flank model of pancreatic cancer was used to study the effect of the $\mathrm{HbS}+\mathrm{GEM}$ formulation. In Figure 4, mice treated with $\mathrm{HbS}+\mathrm{GEM}$ resulted in the smallest tumor size throughout the study and at the final point. Interestingly, there was a trend toward a decreased tumor size in the group treated only with $\mathrm{HbS}$ compared to the control group. This may indicate that there is some degree of benefit in using solely $\mathrm{HbS}$ on the tumor microenvironment. The precise mechanism cannot be elucidated from the current study although a possible explanation is through decreasing interstitial tumor pressure. It has been shown in a number of studies that decreasing interstitial tumor pressure allows a better therapeutic response [6]. Alternatively, it is possible the $\mathrm{HbS}$ polymerization in the tumor causes direct physical disruption of the tumor cell membranes with a resulting decrease in tumor size. This would be similar in mechanism to the pathophysiology that occurs in patients with sickle cell anemia, where $\mathrm{HbS}$ sickles and causes disruption to red blood cell membranes. One main difference between this proposed therapy mechanism and sickle cell anemia sickling is that with the intratumoral injection, the vast majority of the sickling will occur inside the local tumor leaving healthy tissue intact. Another point that supports the notion that the HbS may have an impact on the tumor independently of the chemotherapy cargo is that the growth rate of the tumors from days 15-19 saw steeper growth curves in the groups where $\mathrm{HbS}$ was not present. It is possible that during this time the tumor is most responsive to changes in intratumoral pressure as mentioned above.

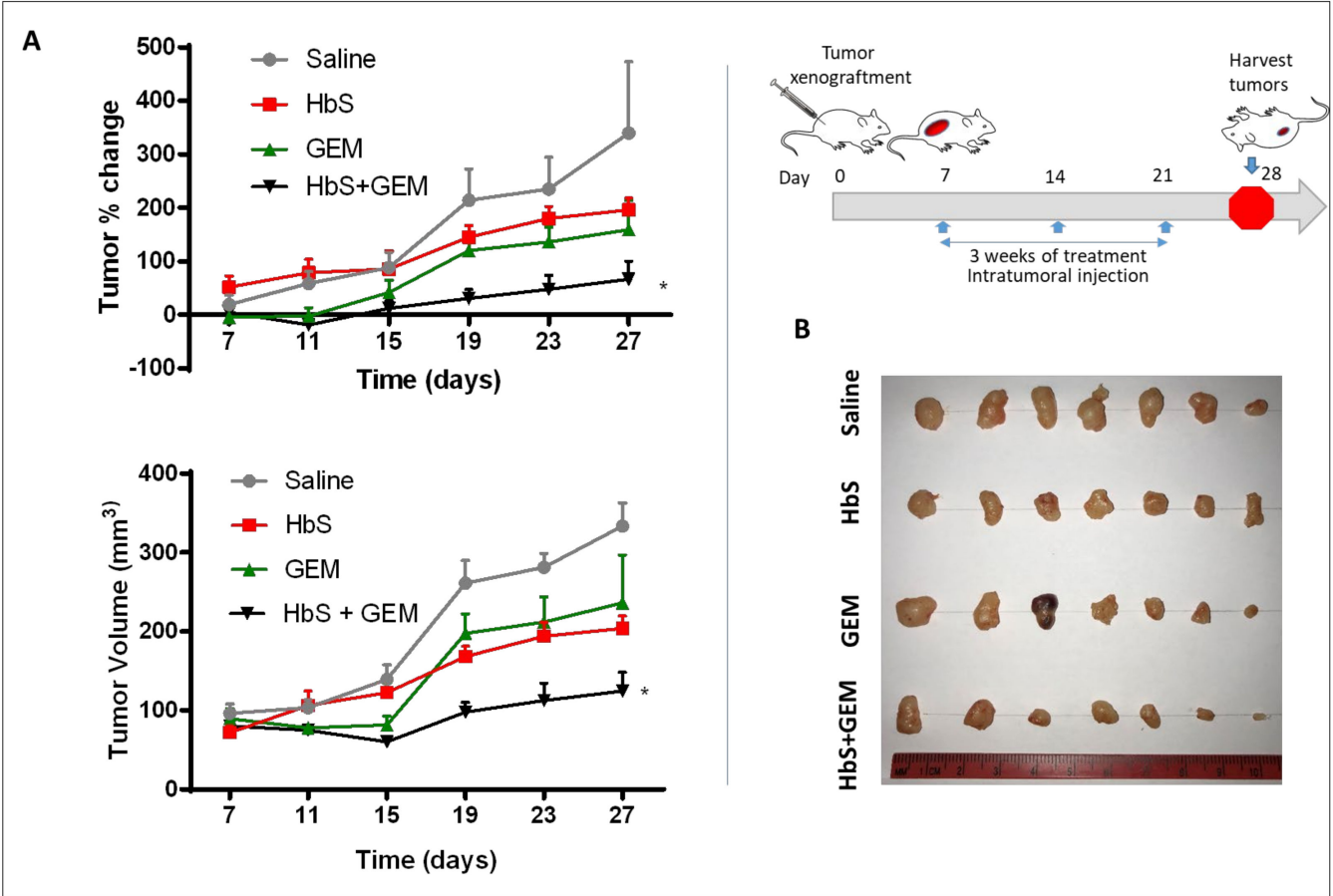

Figure 4. In vivo antitumor efficacy of GEM and $\mathrm{HbS}$ combination. Tumor-growth inhibition in mice treated with saline, $\mathrm{HbS}$, GEM, and $\mathrm{HbS}+\mathrm{GEM}$ by intratumoral injection $(\mathrm{n}=7)$. Tumor percentage change and tumor volume (A). Data given as mean \pm standard deviation $(n=7)$. (B) Photograph of tumors for the four groups of saline, $\mathrm{HbS}, \mathrm{GEM}$, and HbS + GEM. ${ }^{*} p<0.05$; Abbreviations: GEM, gemcitabine; HbS, hemoglobin S.

\section{Discussion}

There are some shortcomings with traditionally developed nanoparticle drug delivery systems due to the complicated chemical synthesis strategies required for manufacturing. Additionally, the drug carrier itself often does not act on the tumor microenvironment and functions solely as a delivery vessel for chemotherapy. Further, there is often a limitation of biocompatibility which can potentially lead to a decreased drug accumulation and 
a subsequent limited tumor response, as the immune system functions to clear out the foreign particles. Finally, the concept of exploiting $\mathrm{pH}$ as a chemotherapy release trigger mechanism has been studied by others in nanotechnology drug delivery platforms that include hydrogels [18-21], as well as others. While this has worked in preclinical models, only incremental progress has been made translating this to clinical success. Little has been investigated on utilizing a mechanism of simultaneously exploiting both the acidic and hypoxic tumor environment for drug delivery.

In this proof-of-concept manuscript we attempted to address these problems of nanoparticle drug carriers mentioned above. The HbS + GEM complex we have developed takes inspiration from the pathological hemoglobin molecule, HbS, which is known to polymerize in humans with sickle cell disease in conditions that are hypoxic and acidic. The simplicity of manufacturing this complex helped mitigate the time-consuming chemical synthesis process and allowed a rapid validation with in vitro and in vivo studies, as well as could lead to easing of the scaling up process required for clinical trials in the future. The simple design of this complex also allows for the possible creation of other hemoglobindrug combination systems that may be useful in other cancers beyond pancreatic cancer, which was experimentally tested in this study. Importantly, the design of this system in which both the drug carrier itself and the drug have a specific antitumor purpose is a critical component. The HbS molecule naturally polymerizes in the same tumor microenvironment that supports tumor cell growth and evasion of chemotherapy. The development of a carrier that can physically disrupt the tumor microenvironment can add synergistic tumor toxicity to the cargo chemotherapy. Additionally, because the HbS differs only by one amino acid from the ubiquitously found natural hemoglobin molecule, we would expect some degree of immune privilege status, helping it to evade the immune system. Additional studies are needed to determine the toxicity of this system, but it is anticipated that the toxicity will be mitigated in healthy areas of the body that are appropriately oxygenated and at a physiologic $\mathrm{pH}$.

This study highlights the potential of a novel bioinspired method of using the selfpolymerizing ability of $\mathrm{HbS}$ together with GEM to improve local delivery in a complex tumor microenvironment. We have designed this therapy to be simply produced and locally delivered intratumorally through the use of endoscopic ultrasound. In vivo intratumoral distribution revealed that $\mathrm{HbS}$ improved the diffusion of small molecules in the tumor microenvironment. Importantly, the delivery of $\mathrm{HbS}$ and GEM acted together in reducing tumor size. Ultimately, this approach could possibly further reduce tumor burden in patients with locally advanced pancreatic cancer and potentially lengthen survival.

Author Contributions: Conceptualization, A.G. and A.P.R.; methodology, A.G. and A.P.R.; investigation, A.G., H.H., R.F. and A.P.R.; writing-original draft preparation, A.G.; formal analysis, A.G., H.H., R.F. and A.P.R.; writing-review and editing, H.H., R.F., J.L., J.K.V., R.J. (Rohan Joshi), P.G., R.J. (Rohan Jeyarajah) and A.P.R.; funding acquisition, J.K.V. and A.P.R.; resources, J.K.V. and A.P.R.; visualization, A.P.R.; supervision, A.P.R. All authors have read and agreed to the published version of the manuscript.

Funding: Research reported in this publication was supported by the intramural seed grantUNTHSC (PI-A.P.R.), the Summerfield G. Roberts Foundation (PI-A.P.R.), National Institute on Minority Health and Health Disparities of the National Institutes of Health under award number U54MD006882 (PI-J.K.V.). The content is solely the responsibility of the authors and does not necessarily represent the official views of the National Institutes of Health.

Institutional Review Board Statement: Animal procedures were performed according to protocols approved by the IACUC at the University of North Texas Health Science Center, Texas, USA (approved number of 2018-0007, dated: 9 March 2018). All procedures related to animal experiments were also performed in accordance with the guidelines established by the Association for Assessment and Accreditation of Laboratory Animal Care International (AAALAC International).

Informed Consent Statement: Not applicable. 
Data Availability Statement: The data presented in this study are available on request from the corresponding author.

Acknowledgments: We would like to acknowledge Khalid Timani for providing the hypoxia incubation facilities for the spectrum analysis.

Conflicts of Interest: The authors declare no conflict of interest. Reata Pharmaceuticals Inc. had no role in the design of the study; in the collection, analyses, or interpretation of data; in the writing of the manuscript, and in the decision to publish the results.

\section{References}

1. Hidalgo, M. Pancreatic cancer. N. Engl. J. Med. 2010, 362, 1605-1617. [CrossRef] [PubMed]

2. Willett, C.G.; Czito, B.G.; Bendell, J.C.; Ryan, D.P. Locally Advanced Pancreatic Cancer. J. Clin. Oncol. 2005, $23,4538-4544$. [CrossRef] [PubMed]

3. Warshaw, A.L.; Fernandez-del Castillo, C. Pancreatic Carcinoma. N. Engl. J. Med. 1992, 326, 455-465. [CrossRef] [PubMed]

4. Von Hoff, D.D.; Ervin, T.; Arena, F.P.; Chiorean, E.G.; Infante, J.; Moore, M.; Seay, T.; Tjulandin, S.A.; Ma, W.W.; Saleh, M.N.; et al. Increased survival in pancreatic cancer with nab-paclitaxel plus gemcitabine. N. Engl. J. Med. 2013, 369, 1691-1703. [CrossRef] [PubMed]

5. $\quad$ Lee, G.Y.; Qian, W.P.; Wang, L.; Wang, Y.A.; Staley, C.A.; Satpathy, M.; Yang, L. Theranostic Nanoparticles with Controlled Release of Gemcitabine for Targeted Therapy and MRI of Pancreatic Cancer. ACS Nano 2013, 7, 2078-2089. [CrossRef]

6. Ariffin, A.B.; Forde, P.F.; Jahangeer, S.; Soden, D.M.; Hinchion, J. Releasing pressure in tumors: What do we know so far and where do we go from here? A review. Cancer Res. 2014, 74, 2655-2662. [CrossRef]

7. Ryan Heather, E.; Poloni, M.; McNulty, W.; Elson, D.; Gassmann, M.; Arbeit, J.M.; Johnson, R.S. Hypoxia-inducible factor-1 $\alpha$ is a positive factor in solid tumor growth. Cancer Res. 2000, 60, 4010-4015.

8. Wilson, W.R.; Hay, M.P. Targeting hypoxia in cancer therapy. Nat. Rev. Cancer 2011, 11, 393-410. [CrossRef] [PubMed]

9. Mandal, A.K.; Mitra, A.; Das, R. Sickle Cell Hemoglobin, Vertebrate and Invertebrate Respiratory Proteins, Lipoproteins and Other Body Fluid Proteins; Springer: Berlin, Germany, 2020; pp. 297-322.

10. Hebbel, R.P.; Vercellotti, G.M. Pathobiology of Sickle Cell Disease. In Hematology: Basic Principles and Practice, 7th ed.; Hoffman, R., Ed.; Elsevier Inc.: Amsterdam, The Netherlands, 2017; pp. 571-583.

11. Takakura, K.; Koido, S. Direct therapeutic intervention for advanced pancreatic cancer. World J. Clin. Oncol. 2015, 6, 216-219. [CrossRef] [PubMed]

12. Patskovska, L.N.; Patskovsky, Y.V.; Almo, S.C.; Hirsch, R.E. COHbC and COHbS crystallize in the R2 quaternary state at neutral $\mathrm{pH}$ in the presence of PEG 4000. Acta Crystallogr. Sect. D 2005, 61, 566-573. [CrossRef] [PubMed]

13. Harrington, D.J.; Adachi, K.; Royer, W.E. The high resolution crystal structure of deoxyhemoglobin S11Edited by K. Nagai. J. Mol. Biol. 1997, 272, 398-407. [CrossRef] [PubMed]

14. Morris, G.M.; Huey, R.; Lindstrom, W.; Sanner, M.F.; Belew, R.K.; Goodsell, D.S.; Olson, A.J. AutoDock4 and AutoDockTools4: Automated docking with selective receptor flexibility. J. Comput. Chem. 2009, 30, 2785-2791. [CrossRef] [PubMed]

15. Trott, O.; Olson, A.J. AutoDock Vina: Improving the speed and accuracy of docking with a new scoring function, efficient optimization, and multithreading. J. Comput. Chem. 2010, 31, 455-461. [CrossRef] [PubMed]

16. Pettersen, E.F.; Goddard, T.D.; Huang, C.C.; Couch, G.S.; Greenblatt, D.M.; Meng, E.C.; Ferrin, T.E. UCSF Chimera-A visualization system for exploratory research and analysis. J. Comput. Chem. 2004, 25, 1605-1612. [CrossRef] [PubMed]

17. Louderback, J.G.; Ballas, S.K.; Kim-Shapiro, D.B. Sickle Hemoglobin Polymer Melting in High Concentration Phosphate Buffer. Biophys. J. 1999, 76, 2216-2222. [CrossRef]

18. Hu, X.; Wei, W.; Qi, X.; Yu, H.; Feng, L.; Li, J.; Wang, S.; Zhang, J.; Dong, W. Preparation and characterization of a novel pH-sensitive Salecan-g-poly (acrylic acid) hydrogel for controlled release of doxorubicin. J. Mater. Chem. B3 2015, 3, $2685-2697$. [CrossRef] [PubMed]

19. Yan, G.; Wang, J.; Hu, L.; Wang, X.; Yang, G.; Fu, S.; Cheng, X.; Zhang, P.; Tang, R. Stepwise targeted drug delivery to liver cancer cells for enhanced therapeutic efficacy by galactose-grafted, ultra-pH-sensitive micelles. Acta Biomater. 2017, 15, 363-373. [CrossRef] [PubMed]

20. Ranalli, A.; Santi, M.; Capriotti, L.; Voliani, V.; Porciani, D.; Beltram, F.; Signore, G. Peptide-based stealth nanoparticles for targeted and pH-triggered delivery. Bioconjugate Chem. 2017, 28, 627-635. [CrossRef] [PubMed]

21. Meng, F.; Zhong, Y.; Cheng, R.; Deng, C.; Zhong, Z. pH-sensitive polymeric nanoparticles for tumor-targeting doxorubicin delivery: Concept and recent advances. Nanomedicine 2014, 9, 487-499. [CrossRef] [PubMed] 